\title{
Effect of Remote Ischemic Preconditioning on Troponin I in CABG
}

\author{
Fatemeh Javaherforoosh Zadeh,, Mohsen Moadeli, ${ }^{2,}{ }^{*}$ Mansoor Soltanzadeh, ${ }^{3}$ and Farahzad
}

\begin{abstract}
Janatmakan ${ }^{2}$
${ }^{1}$ Associate Professor of Anesthesia, Department of Anesthesia, Ahvaz Anesthesiology and Pain Research Center, Ahvaz Jundishapur University of Medical Sciences, Ahvaz, Iran ${ }^{2}$ Assistant Professor of Anesthesia, Department of Anesthesia, Ahvaz Anesthesiology and Pain Research Center, Ahvaz Jundishapur University of Medical Sciences, Ahvaz, Iran ${ }^{3}$ Professor of Anesthesia, Department of Anesthesia, Ahvaz Anesthesiology and Pain Research Center, Ahvaz Jundishapur University of Medical Sciences, Ahvaz, Iran

"Corresponding author: Mohsen Moadeli, Anesthesia Department, Imam Khomeini Hospital, Pain Research Center, Ahvaz Jundishapur University of Medical Sciences, Ahvaz, Iran. Tel: +98-9173160725, +98-6133743037, E-mail: moadelimohsen@yahoo.com
\end{abstract}

Received 2017 April 04; Revised 2017 May 04; Accepted 2017 August 07.

\begin{abstract}
Background: Elective open heart surgery is associated with troponin release in some cases due to myocyte necrosis.

Objectives: The aim of this study was to measure cardiac troponin I (cTnI) preoperatively in elective CABG after remote ischemic preconditioning.

Methods: Twenty-eight patients were selected for elective CABG. They were randomized to receive remote ischemic preconditioning (induced by three 5-min cycles of inflation with a pneumatic tourniquet and 5-min deflation between inflation episodes as reperfusion).

Outcomes: Primary outcomes were cardiac troponin I levels at 6 and 24 hours after the procedure, and the secondary outcomes included creatine phosphokinase, lactate dehydrogenase, and serum creatinine levels. Hemodynamic changes were evaluated between the treatment and control groups.

Results: Cardiac troponin I at 6 hours after preconditioning was significantly lower compared to the control group $(\mathrm{P}=0.036)$, and after 24 hours, there was still a significant difference between the two groups $(\mathrm{P}<0.05)$.

Conclusions: Remote ischemic preconditioning reduces ischemic biomarkers during coronary artery bypass graft and attenuates procedure-related cardiac troponin I release and eventually reduces cardiovascular events such as myocardial infarction, chest pain, and hemodynamic changes after cardiac surgery.
\end{abstract}

Keywords: Cardiac Surgery, Remote Ischemic Preconditioning, Cardiac Outcomes

\section{Background}

Elective open heart surgery is associated with troponin release in some cases (1). Troponin elevation is associated with necrosis and damage to cardiac cells (myocytes), and it is a sensitive and specific blood marker for ischemia and reperfusion cycles (1). In some studies, elevation of cardiac troponin 1 (cTnI) has been associated with cardiovascular events such as arrhythmia, hemodynamic changes, and perioperative myocardial infarction (2). One recognized approach for reducing myocardial damage is ischemic preconditioning in cardiovascular surgery, which is a cardioprotective procedure with brief episodes of myocardial ischemia and reperfusion cycles before a potentially highrisk cardiac surgery (3). Recent studies suggest that a less invasive procedure may be more helpful with remote ischemic preconditioning (RIPC) in cardiovascular surgery. RIPC was first introduced by Przyklenk in laboratory animals, where he demonstrated that brief ischemia of the left circumflex artery could modify the unfavorable effects of ischemia in the left anterior descending artery (4).

RIPC can be achieved by noninvasive inflation and de- flation of a standard blood pressure cuff or pneumatic tourniquet on the upper or lower extremities to induce brief ischemia and reperfusion, a procedure that causes injury in patients undergoing open cardiac surgery. Anaerobic metabolism and decreased ATP production occur due to ischemia, and there is insufficient energy for cell membrane pump activity, antioxidant defense, and homeostasis, which leads to cell death. On the other hand, in the reperfusion cycle, sudden entry of oxygen which is called oxygen influx leads to synthesis of reactive oxygen species and causes reperfusion syndrome (5).

\section{Objectives}

The aim of this study was to evaluate the capability of RIPC to reduce cTnI levels after elective CABG.

\section{Methods}

We performed a randomized clinical trial on patients scheduled for CABG during January and Febru-

Copyright ( $)$ 2017, Anesthesiology and Pain Medicine. This is an open-access article distributed under the terms of the Creative Commons Attribution-NonCommercial 4.0 International License (http://creativecommons.org/licenses/by-nc/4.0/) which permits copy and redistribute the material just in noncommercial usages, provided the original work is properly cited. 
ary 2017, which included 28 patients referred for onpump open-heart surgery. This research was supported by the Pain Research Center, Ahvaz Jundishapur University of Medical Sciences, Ahvaz, Iran, under proposal No. IR.AJUMS.REC.1395.501. All patients provided written informed consent. Patients were excluded from consideration for the following reasons: more than 70 or less than 20 years old, ASA class 4 or more, elevated baseline cTnI, myocardial infarction in preceding 4 weeks, ejection fraction less than $35 \%$, renal dysfunction, arteriovenous fistula, lymph edema, and severe hepatic or endocrine dysfunction. Patients were randomly selected to undergo openheart surgery with (14 patients) or without (control, 14 patients) RIPC (Randomization method was random permuted block sizes 4 ).

There are different protocols for RIPC. In this study, RIPC consisted of three 5-min cycles of cuff inflation on the right arm up to $200 \mathrm{mmHg}$, which is called the ischemia phase, and 5-min cuff deflation between inflation cycles, which is called the reperfusion phase. The inflation and deflation phases were induced with a pneumatic tourniquet placed on the right upper arm. Control patients had a deflated cuff placed on the right upper arm for 30 minutes. This process was applied after induction of anesthesia and baseline chemistries.

Standard anesthetic, surgical, and cardiopulmonary bypass ( $\mathrm{CPB}$ ) techniques were employed. Anesthesia was induced with $0.03 \mathrm{mg} / \mathrm{kg}$ midazolam, $0.1 \mu \mathrm{g} / \mathrm{kg}$ sufentanyl, $1 \mathrm{mg} / \mathrm{kg}$ ketamine, and $0.5 \mathrm{mg} / \mathrm{kg}$ cisatracurium. Anesthesia was maintained with $0.1 \mathrm{mg} / \mathrm{kg} / \mathrm{h}$ midazolam, $0.1 \mu / \mathrm{kg} / \mathrm{h}$ sufentanyl, $0.1 \mathrm{mg} / \mathrm{kg} / \mathrm{h}$ cisatracurium, and $0.6 \%$ isoflurane in 50\% oxygen. All operations were performed under normothermic CPB, and antegrade cold crystalloid cardioplegia (St. Thomas solution) was used every 20 - 25 minutes until completion. After reconstruction of all the grafts, $\mathrm{CPB}$ was gradually discontinued and intravenous protamine was administered to reverse heparin anticoagulant effects.

Central venous pressure and mean arterial pressure were recorded in all patients before, 6 and 24 hours after RIPC.

The impact of RIPC on myocardial injury was assessed by analyzing biochemical markers. cTnI, creatine phosphokinase (CPK) and lactate dehydrogenase (LDH) were determined in blood samples taken preoperatively and at the time points of 6 and 24 hours after RIPC. Plasma cTnI was determined quantitatively using the Troponin-I test system (Monobind Inc., Lake Forest, CA, USA) with a normal range $<1.3 \mathrm{ng} / \mathrm{mL}$.

Statistical methods: Statistical analyses were performed using SPSS 22. Analysis of variance or t-test was used to compare continuous variables, and chi-square test was used to compare categorical variables. Qualitative data are expressed in absolute numbers (percentage), and quantitative data are presented as the mean and standard deviation. $\mathrm{P}<0.05$ was considered statistically significant.

\section{Results}

Twenty-eight patients were approached for study inclusion. There were no statistically significant differences between the two groups according to demographic and clinical characteristics (Table 1). The evaluation of hemodynamic data indicated that systolic blood pressure was not significantly different compared to the control group after 6 hours of RIPC [141.13 (137.03; 145.23) versus 146 (140.98; 151.01) $\mathrm{mmHg} ; \mathrm{P}=1$ ], but there was a significant difference after 24 hours [137.73 (133.19;142.26) versus 147 (143.42; 151.03); $\mathrm{P}=0.025]$. Diastolic blood pressure showed no significant difference between the RIPC and control groups after $6 \mathrm{~h}[81.93(78.34 ; 85.51)$ versus $83.38(79.15 ; 87.61) \mathrm{mmHg}$; $\mathrm{P}=1$ ], but there was a significant difference after 24 hours [77.73 (74.8; 80.66) versus $86(82.22 ; 89.77) \mathrm{mmHg} ; \mathrm{P}=0.011$ ] Mean arterial pressure in the RIPC group compared to the control group after $6 \mathrm{~h}$ showed no significant difference [101.4 (97.79; 105) versus 103.84 (99.59; 108.09) $\mathrm{mmHg}$ P = 0.198]. After 24 hours, mean arterial pressure was significantly lower in the RIPC group compared to the control group [97.53 (94.31; 100.74) versus 106.07 (102.48; 109.67) $\mathrm{mmHg} ; \mathrm{P}=0.008]$. The assessment data indicated that central venous pressure was not lower in the RIPC group compared to the controls after 6 hours [5.33 (4.85; 5.81) versus 5.92 (5.52; 6.31) $\mathrm{mmHg} ; \mathrm{P}=0.937]$, but that it was significantly lower after 24 hours [5.13 $(4.61 ; 5.65)$ versus 6.84 (6.28; 7.4) mmHg; P $<0.05]$.

Table 1. Baseline Characteristics of the Patient

\begin{tabular}{|c|c|c|}
\hline Variable & RIPC & Control \\
\hline No. of patients & 14 & 14 \\
\hline Sex (male/female) & $8 / 6$ & $10 / 4$ \\
\hline Age, $y$ & $57.7 \pm 10.2$ & $57.2 \pm 7.04$ \\
\hline BMI, $\mathrm{kg} / \mathrm{m}^{2}$ & $27.5 \pm 2.04$ & $27.7 \pm 1.94$ \\
\hline LVEF, \% & $51.2 \pm 3.11$ & $51.4 \pm 3.81$ \\
\hline
\end{tabular}

Abbreviations: BMI, body mass index; LV EF, left ventricular ejection fraction; RIPC, remote ischemic preconditioning.

Blood chemistries indicating myocardial injury showed that the baseline cTnI level was in the normal range of the assay $(<1.3 \mathrm{ng} / \mathrm{mL})$ in both groups. Plasma cTnI was significantly lower 6 hours after surgery in the RIPC group compared to the control group [ $0.29 \pm 0.07$ versus $0.70 \pm 0.11 \mathrm{ng} / \mathrm{mL}$ for the controls; $\mathrm{P}=0.036]$ ]. 
After 24 hours, the cTnI level was significantly lower in the RIPC group compared to the control group [0.16 \pm 0.08 versus $1.22 \pm 0.18 \mathrm{ng} / \mathrm{mL}$ for the controls; $\mathrm{P}<0.05$ ]. Similar changes were seen in serum CPK (184.66 \pm 6.00 versus $225.38 \pm 8.46 ; \mathrm{P}=0.36)$ at $6 \mathrm{~h}$ and $(179.33 \pm 5.85$ versus236.61 $\pm 8.06 ; \mathrm{P}<0.05) 24$ hours after RIPC. Changes in serum creatinine $(0.90 \pm 0.04$ versus $1.17 \pm 0.04 ; \mathrm{P}=$ 0.000 ) were detected significant 24 hours after surgery but not significant at $6 \mathrm{~h}$. There was no significant change in serum LDH (Table 2). The findings suggested that there was a relationship between CTnI and CPK elevation and clinical outcomes.

\section{Discussion}

This study demonstrated that RIPC administered by brief arm ischemia-reperfusion episodes stimulates CABGrelated troponin release in patients undergoing elective CABG. RIPC before cardiac surgery seems to improve clinical outcomes after surgery $(6,7)$. Most studies have shown that open-heart surgery-related cTnI elevation is associated with poor outcome (7). An increase in cTnI level more than 5 -fold baseline levels is a predictor of death, revascularization, and myocardial infarction (8-10). When the post open-heart surgery cTnI concentration is elevated up to 3 times compared to the baseline, myocardial infarction specifically relates to surgery as the cause (11). The most recognized mechanisms of $\mathrm{cTnI}$ release is microembolization of plaque and side-branch occlusion (11). In Doppler studies, there are microembolic signals during cardiac surgery associated with cTnI release (12). Birnbaum and colleagues demonstrated that RIPC in the rabbit reduced infarct size by about $60 \%$ compared to the control group (12). RIPC shows a biphasic model for myocardial protection (13). The first phase, called the early classic phase, starts a few minutes to 2 hours after RIPC and stimulates mitochondrial ATP-sensitive potassium channels. The second phase, called the delayed phase, occurs at 24 to 72 hours after RIPC, and it may be the cause of the expression of genes that inhibits the proinflammatory response to ischemic/reperfusion injury (13). In some studies, no myocardial protection by RIPC was reported, such as that of Porto and colleagues. Lee et al. studied RIPC effects in pulmonary hypertensive infants undergoing ventricular septal defect surgery and found that the RIPC could not produce any substantial myocardial or pulmonary protection (14). The RIPC protection mechanism for myocardium against ischemia and reperfusion injury is unclear (15). But some suggested pathways emphasize the release of substances from the remote organ that activate intracellular signaling cascades in the myocardium (16). In recent decades, RIPC has received more attention since it can reduce injury to the myocardium after brief episodes of myocardial ischemia (17). Since this procedure is non-invasive and safe, it may be applicable in patients with coronary disease (17).

We demonstrated in this study that RIPC induced by short ischemia and reperfusion episodes to the arm was able to control systolic, diastolic, and mean arterial pressure compared to the control groups. The most probable cause of better control of blood pressure has been reported in some studies to be associated with mediators such as circulating opioids, calcitonin, endocannabinoids, bradykinin, and adenosine (18).

Release of cardiac biomarkers during heart surgery occurs as a result of ischemia and reperfusion injury (18). Biomarkers of myocardial injury, such as LDH, CPK, and cTnI, have been studied in several trials and have been reported as results of injuries in cardiac surgeries (18). Kidney protection in patients undergoing cardiovascular surgery is controversial. In some studies such as that of D'Ascenzo et al. a meta-analysis that examined the effect of RIPC in patients undergoing cardiovascular interventions, the authors found no significant differences in serum creatinine between the RIPC group and control group (19). On the other hand, a meta-analysis conducted by Alreja et al. demonstrated significant differences in serum creatinine level between the RIPC and control groups (19). In this study, we showed that RIPC reduced myocardial damage in patients undergoing open -heart surgery. There were significant differences in plasma levels of biochemical markers of myocardial damage, namely CTnI, CPK, and LDH, at 6 and 24 hours after the procedure between the RIPC group and control group.

Our study had potential limitations. Although there were enough patients undergoing cardiac surgery for the RIPC procedure, we were unable to do a whole blindness for the procedure.

Conclusions: We conclude that RIPC may reduce myocardial injury in patients undergoing open-heart surgery.

\section{Acknowledgments}

This research was supported by the Pain Research Center, Ahvaz Jundishapur University of Medical Sciences, Ahvaz, Iran, with proposal number IR.AJUMS.REC.1395.501 and IRCT2016120431226N1. The authors appreciate the assistance of the patients who participated in the study. Dr. A. Leyva (USA) provided English editing of the manuscript. 
Table 2. Biomarkers After Surgery

\begin{tabular}{|c|c|c|c|c|c|c|c|}
\hline Marker & Group & Before RIPC & P Value & $6 \mathrm{~h}$ after RIPC & P Value & $24 \mathrm{~h}$ after RIPC & PValue \\
\hline \multirow{2}{*}{ Troponin I, ng/mL } & RIPC & $0.88 \pm 0.10$ & \multirow{2}{*}{0.65} & $0.29 \pm 0.07$ & \multirow{2}{*}{$0.036^{\mathrm{a}}$} & $0.16 \pm 0.08$ & \multirow{2}{*}{$<0.05^{\mathrm{a}}$} \\
\hline & Control & $0.33 \pm 0.11$ & & $0.70 \pm 0.11$ & & $1.22 \pm 0.18$ & \\
\hline \multirow{2}{*}{ CPK, U/L } & RIPC & $190.40 \pm 5.05$ & \multirow{2}{*}{0.628} & $184.66 \pm 6.00$ & \multirow{2}{*}{$0.036^{\mathrm{a}}$} & $179.33 \pm 5.85$ & \multirow{2}{*}{$<0.05^{\mathrm{a}}$} \\
\hline & Control & $210.61 \pm 8.55$ & & $225.38 \pm 8.46$ & & $236.61 \pm 8.06$ & \\
\hline \multirow{2}{*}{ LDH, U/L } & RIPC & $351.40 \pm 25.05$ & \multirow{2}{*}{1} & $344.86 \pm 25.75$ & \multirow{2}{*}{1} & $335.53 \pm 26.82$ & \multirow{2}{*}{1} \\
\hline & Control & $354.61 \pm 24.77$ & & $376.23 \pm 24.99$ & & $391.07 \pm 25.33$ & \\
\hline \multirow{2}{*}{ Creatinine, mg/dL } & RIPC & $0.99 \pm 0.04$ & \multirow{2}{*}{1} & $0.93 \pm 0.05$ & \multirow{2}{*}{0.1} & $0.90 \pm 0.04$ & \multirow{2}{*}{0.000} \\
\hline & Control & $1.01 \pm 0.05$ & & $1.10 \pm 0.03$ & & $1.17 \pm 0.04$ & \\
\hline
\end{tabular}

Abbreviations: CPK, creatine phosphokinase; $\mathrm{LDH}$, lactate dehydrogenase.

${ }^{\mathrm{a}}$ Significant.

\section{Footnotes}

Authors' Contribution: Study concept and design: Fatemeh Javaherforoosh Zadeh and Mohsen Moadeli; data collection: Mohsen Moadeli.

Funding/Support: Pain research center, Ahvaz Jundishapur University of Medical Sciences, Ahvaz, Iran

\section{References}

1. Hoole SP, Heck PM, Sharples L, Khan SN, Duehmke R, Densem CG, et al. Cardiac Remote Ischemic Preconditioning in Coronary Stenting (CRISP Stent) Study: a prospective, randomized control trial. Circulation. 2009;119(6):820-7. doi: 10.1161/CIRCULATIONAHA.108.809723. [PubMed: 19188504].

2. Kizer JR, Muttrej MR, Matthai WH, McConnell J, Nardone H, Sonel AF, et al. Role of cardiac troponin $\mathrm{T}$ in the long-term risk stratification of patients undergoing percutaneous coronary intervention. Eur Heart J. 2003;24(14):1314-22. [PubMed:12871688].

3. Lucchinetti E, Bestmann L, Feng J, Freidank H, Clanachan AS, Finegan BA, et al. Remote ischemic preconditioning applied during isoflurane inhalation provides no benefit to the myocardium of patients undergoing on-pump coronary artery bypass graft surgery: lack of synergy or evidence of antagonism in cardioprotection? Anesthesiology. 2012;116(2):296-310. doi: 10.1097/ALN.0b013e318242349a. [PubMed: 22222469].

4. Lattouf OM, Thourani VH, Kilgo PD, Halkos ME, Baio KT, Myung $\mathrm{R}$, et al. Influence of on-pump versus off-pump techniques and completeness of revascularization on long-term survival after coronary artery bypass. Ann Thorac Surg. 2008;86(3):797-805. doi: 10.1016/j.athoracsur.2008.04.065. [PubMed:18721564].

5. Hausenloy DJ, Candilio L, Evans R, Ariti C, Jenkins DP, Kolvekar $S$, et al. Remote Ischemic Preconditioning and Outcomes of Cardiac Surgery. N Engl J Med. 2015;373(15):1408-17. doi: 10.1056/NEJMoa1413534. [PubMed: 26436207].

6. Lomivorotov VV, Shmyrev VA, Nepomnyaschih VA, Ponomarev DN, Knyazkova LG, Lomivorotov VN, et al. Remote ischaemic preconditioning does not protect the heart in patients undergoing coronary artery bypass grafting. Interact Cardiovasc Thorac Surg. 2012;15(1):18-22. doi:10.1093/icvts/ivs118. [PubMed: 22493101].

7. Pell T], Baxter GF, Yellon DM, Drew GM. Renal ischemia preconditions myocardium: role of adenosine receptors and ATP-sensitive potassium channels. Am J Physiol. 1998;275(5 Pt 2):H1542-7. [PubMed: 9815059].
8. Shimizu M, Tropak M, Diaz RJ, Suto F, Surendra H, Kuzmin E, et al. Transient limb ischaemia remotely preconditions through a humoral mechanism acting directly on the myocardium: evidence suggesting cross-species protection. Clin Sci (Lond). 2009;117(5):191-200. doi: 10.1042/CS20080523. [PubMed: 19175358].

9. Choi YS, Shim JK, Kim JC, Kang KS, Seo YH, Ahn KR, et al. Effect of remote ischemic preconditioning on renal dysfunction after complex valvular heart surgery: a randomized controlled trial. J Thorac Cardiovasc Surg. 2011;142(1):148-54. doi: 10.1016/j.jtcvs.2010.11.018. [PubMed: 21272897].

10. Karuppasamy P, Chaubey S, Dew T, Musto R, Sherwood R, Desai J, et al. Remote intermittent ischemia before coronary artery bypass graft surgery: a strategy to reduce injury and inflammation? Basic Res Cardiol. 2011;106(4):511-9. doi: 10.1007/s00395-011-0185-9. [PubMed: 21544683].

11. Hu S, Dong HL, Li YZ, Luo ZJ, Sun L, Yang QZ, et al. Effects of remote ischemic preconditioning on biochemical markers and neurologic outcomes in patients undergoing elective cervical decompression surgery: a prospective randomized controlled trial. J Neurosurg Anesthesiol. 2010;22(1):46-52. doi:10.1097/ANA.0b013e3181c572bd. [PubMed: 19996767].

12. D'ascenzo F, Cavallero E, Moretti C, Omede P. , Sciuto F, Rahman IA et al. Remote ischaemic preconditioning in coronary artery bypass surgery: a meta-analysis. Heart. 2012;98(17):1267-71.

13. Abdul-Ghani S, Heesom KJ, Angelini GD, Suleiman MS. Cardiac phosphoproteomics during remote ischemic preconditioning: a role for the sarcomeric Z-disk proteins. Biomed Res Int. 2014;2014:767812. doi: 10.1155/2014/767812. [PubMed: 24795895].

14. Lee JH, Park YH, Byon HJ, Kim HS, Kim CS, Kim JT. Effect of remote ischaemic preconditioning on ischaemic-reperfusion injury in pulmonary hypertensive infants receiving ventricular septal defect repair. BrJAnaesth. 2012;108(2):223-8. doi:10.1093/bja/aer388. [PubMed: 22157844].

15. Oxman T, Arad M, Klein R, Avazov N, Rabinowitz B. Limb ischemia preconditions the heart against reperfusion tachyarrhythmia. Am J Physiol. 1997;273(4 Pt 2):H1707-12. [PubMed: 9362234].

16. Porto I, Blackman DJ, Nicolson D, Niccoli G, Kahn FZ, Ormerod O, et al. What is the incidence of myocardial necrosis in elective patients discharged on the same day following percutaneous coronary intervention? Heart. 2004;90(12):1489-90. doi: 10.1136/hrt.2003.027672. [PubMed: 15547042].

17. Zhu SB, Liu Y, Zhu Y, Yin GL, Wang RP, Zhang Y, et al. Remote preconditioning, perconditioning, and postconditioning: a comparative study of their cardio-protective properties in rat models. Clinics (Sao Paulo). 2013;68(2):263-8. [PubMed: 23525325].

18. Kloner RA. Clinical application of remote ischemic preconditioning. Circulation. 2009;119(6):776-8. doi: 10.1161/CIRCULATIONAHA.108.832832. [PubMed: 19221230]. 
19. Li L, Li G, Yu C, Li Y. The role of remote ischemic preconditioning on postoperative kidney injury in patients undergoing cardiac and vas- cular interventions: a meta-analysis. J Cardiothorac Surg. 2013;8:43. doi: 10.1186/1749-8090-8-43. [PubMed: 23497625]. 